\title{
Efeitos Tardios do Bullying e Transtorno de Estresse Pós-Traumático: Uma Revisão Crítica
}

\author{
Paloma Pegolo de Albuquerque ${ }^{1}$ \\ Lúcia Cavalcanti de Albuquerque Williams \\ Sabrina Mazo D’Affonseca \\ Universidade Federal de São Carlos
}

\begin{abstract}
RESUMO - O Transtorno de Estresse Pós-Traumático (TEPT) é considerado o principal transtorno psiquiátrico associado à violência. A fim de analisar a possível relação entre bullying e o desenvolvimento tardio de sintomas de TEPT, o objetivo do presente trabalho foi o de identificar e organizar a produção cientifica da área. Para tanto, foi realizada uma revisão bibliográfica na literatura considerando livros e artigos publicados, bem como pesquisa eletrônica em bases de dados. A análise das pesquisas encontradas indicou que, apesar de os resultados apontarem uma relação entre TEPT e bullying, tanto na infância quanto a longo prazo, não há dados suficientes que explicitem como se dá essa relação. Contudo, há evidências de que alguns indivíduos que sofrem vitimização por bullying possam apresentar maior vulnerabilidade para desenvolver TEPT.
\end{abstract}

Palavras-chave: bullying, TEPT, violência, trauma

\section{Long Term Effects of Bullying and Posttraumatic Stress Disorder: A Literature Review}

\begin{abstract}
Posttraumatic Stress Disorder (PTSD) has been identified as the main psychiatric disorder associated with violence. This paper had the objective of identifying and organizing current scientific knowledge on long term effects on bullying and the development of PTSD. Thus, a literature review was conducted involving published books and papers on the topic, as well as an electronic database survey. Although studies indicate a relation between PTSD and bullying during childhood and later, there is a lack of research clarifying how this relationship occurs. There is evidence showing that some individuals who are victimized by bullying may present greater vulnerability to develop PTSD.
\end{abstract}

Keywords: bullying, PTSD, violence, trauma

Há muitos tipos de violência na escola, sendo que a vitimização escolar pode ser praticada tanto por pares quanto por professores. As consequências para alunos vitimizados na escola incluem sintomas físicos, doenças psicossomáticas, prejuízos sociais, emocionais e acadêmicos; sendo comuns: a depressão (Bond, Carlin, Thomas, Rubin, \& Patton, 2001), a ansiedade (Fekkes, Pijpers, Fridriks, Vogels, \& Verloove-Vanhorick, 2010) e os sintomas de Transtorno de Estresse Pós-Traumático (TEPT).

A escola tem sido apontada, pela literatura, como um local no qual a violência é cada vez mais comum (Francisco \& Libório, 2009; Furlong, Morrison, \& Jimerson, 2004; Ruotti, Alves, \& Cubas, 2007). São frequentes as agressões entre alunos e funcionários (e vice-versa), os danos contra o patrimônio escolar e, também, a agressão entre pares, conhecida como bullying, sendo tal fenômeno o foco do presente estudo.

\section{O bullying no contexto escolar}

De acordo com Olweus (1991), pesquisador pioneiro sobre violência entre pares na escola, bullying refere-se a ações físicas e sociais negativas que são cometidas intencionalmente,

1 Endereço para correspondência: Rua Guatemala, 166, Vila Brasília, São Carlos, SP. CEP: 13.566-720. E-mail: palomanier@yahoo.com.br repetidamente, ao longo do tempo por uma ou mais pessoas contra um indivíduo que não pode se defender facilmente. Para Craig et al. (2009) e Lamb, Pepler e Craig (2009) bullying é o uso do poder com o intuito de controlar os pares.

O bullying pode ser dividido em quatro categorias: $f i$ sico, que envolve comportamentos como empurrar, bater, chutar; verbal, que inclui ameaçar, xingar; relacional, que se refere a atos que danifiquem o relacionamento entre pares, como exclusão de atividades, propagação de fofocas e mentiras; e o sexual, envolvendo condutas e comentários de natureza sexual (Orpinas \& Horne, 2006). Há ainda o cyberbullying, no qual as agressões são feitas por meio de recursos eletrônicos, como computadores e celulares (Lamb et al., 2009; Trautmann, 2008).

É difícil estimar a prevalência do bullying devido à multiplicidade de definições e diversidade de metodologias empregadas na coleta de dados, que às vezes não consideram todas as categorias de comportamento do fenômeno (Finkelhor, Turner, \& Hamby, 2012; Lien, Green, Welander-Vatn, \& Bjertness, 2009; Orpinas \& Horne, 2006). Além disso, há limitações temporais e culturais, como, por exemplo, a não existência do termo em todas as línguas, havendo, também, diferenças entre os índices de bullying em países diferentes (Campbell, 2004).

De forma geral, os índices mundiais de bullying mostram-se preocupantes. Segundo Orpinas e Horne (2006), 
nos Estados Unidos, 41\% dos estudantes reportaram ter sido vítima de bullying. Cerezo e Ato (2010) encontraram uma prevalência de bullying de $17,4 \%$ na amostra de estudantes espanhóis estudada. No estudo de Craig et al. (2009), comparando estimativas de prevalência de bullying e vitimização em 40 países, contando com a participação de mais de 200.000 estudantes, 26\% da amostra reportou envolvimento com bullying; $10,7 \%$ afirmaram serem agressores; $12,6 \%$ reportaram serem vítimas e 3,6\% relataram serem vítimas/agressores.

Embora o bullying seja um fenômeno muito pesquisado no exterior, o estudo de sua ocorrência na população brasileira começou há menos tempo (Francisco \& Libório, 2009; Neme, Mello, Gazzola, \& Justi, 2008). Os primeiros estudos brasileiros (mencionados a seguir) revelaram que a intimidação física ou psicológica entre pares tem uma prevalência expressiva também entre nossos alunos.

No estudo publicado em 2003 pela Associação Brasileira de Proteção à Infância e à Adolescência (ABRAPIA), $40,5 \%$ dos 5.482 alunos participantes relatou ter tido algum envolvimento direto em situações de bullying (como vítima e/ou agressor). Muitos dos alunos caracterizados como vítimas relataram ter sentimentos negativos como medo, raiva, preocupações e vontade de faltar às aulas (Lopes Neto \& Saavedra, 2003). Na pesquisa de Pinheiro e Williams (2009) com 239 estudantes de três escolas públicas de uma cidade do interior de São Paulo, com idades entre 11 e 15 anos, foi percebida uma alta prevalência de bullying, pois 49\% dos participantes relatou algum envolvimento em bullying. Em contraste, na pesquisa de Francisco e Libório (2009) com 283 alunos de uma cidade do interior paulista, com idade média de 12,82 anos, $37,2 \%$ relataram ter sofrido bullying ou ameaças na escola.

Diversos pesquisadores tem apontado que existem papéis característicos no processo de bullying: agressor - inicia a agressão e é líder; seguidores do agressor, divididos em assistentes (ajudam o agressor) e reforçadores (reforçam os comportamentos do agressor, aplaudindo, rindo e incentivando); expectadores, divididos naqueles que fazem parte do problema (incentivando ou omitindo-se) e nos que fazem parte da solução (ajudando, buscando ajuda); e vítima ou alvo da agressão, que é abusada e assediada sistematicamente pelo agressor (Orpinas \& Horne, 2006). No entanto, esses papéis não são rígidos, podendo haver intercâmbio entre eles, sendo que uma vítima pode se tornar agressor em outra situação (Batsche \& Knoff, 1994).

De acordo com Batsche e Knoff (1994), algumas características dos agressores são: maior força física do que a vítima, comportamento impulsivo, comportamento agressivo, necessidade de dominar os colegas e pouca empatia pelos outros. Trautmann (2008) acrescenta também baixa tolerância à frustração e desafio a autoridades.

Quanto às vítimas, alguns autores dividem-nas em dois tipos: passivas, que costumam ser mais ansiosas, quietas e submissas que as outras crianças (Orpinas \& Horne, 2006); e provocativas, normalmente apresentando comportamento ansioso, irritadiço, tentando vingar-se (Batsche \& Knoff, 1994). Os autores acrescentam, também, que as vítimas costumam desfrutar de baixo status entre os colegas, sendo rejeitadas e mesmo isoladas do grupo (Cerezo \& Ato, 2010;
Scholte, Engels, Overbeek, Kemp, \& Haselager, 2007; Spriggs et al., 2007).

Para Cerezo e Ato (2010), é comum a rejeição social, tanto dos agressores, quanto das vítimas, mas os agressores são percebidos como fortes, enquanto as vítimas são vistas como covardes e acabam sendo mais excluídas das atividades, o que faz com que tais alunos tenham uma percepção da sala de aula como hostil e isoladora. A rejeição social sofrida tanto pelos agressores, quanto pelas vítimas, mostra a grande influência dos pares no processo de bullying (Furlong et al., 2004; Spriggs, Iannotti, Nansel, \& Haynie, 2007).

Segundo Lien et al. (2009) e Batsche e Knoff (1994), há uma tendência geral dos estudantes do sexo masculino sofrerem e aplicarem mais bullying do que as estudantes. $\mathrm{Na}$ pesquisa de Craig et al. (2009), o índice de bullying entre os garotos foi de $23,4 \%$, indo de $8,6 \%$ na Suécia a $45,2 \%$ na Lituânia; e entre garotas foi de $15,8 \%$, variando de $4,8 \%$ na Suécia a 35,8\% na Lituânia. Os tipos de bullying também parecem ser diversos: as garotas reportam mais problemas de relacionamento, exclusão e bullying verbal do que o físico, relatado mais frequentemente pelos meninos (Campbell, 2004; Craig et al., 2009; Craig \& Pepler, 2003; Lien et al., 2009; Lopes-Neto, 2005; Trautmann, 2008).

Spriggs et al. (2007) apontam alguns aspectos que influenciam o comportamento de bullying, como as interações familiares e a postura da escola diante de situações de violência. A escola é um ambiente de socialização ímpar para crianças e adolescentes e, muitas vezes, há falha no papel de oferecer um ambiente seguro, estável e propício à aprendizagem, favorecendo o desenvolvimento pleno dos estudantes. Portanto, é imprescindível que a mesma colabore na prevenção e erradicação do bullying.

Por sua vez, o ambiente familiar pode afetar o comportamento de bullying de múltiplas maneiras. Em primeiro lugar há a imitação de comportamentos agressivos, no caso do autor do fenômeno, por meio da exposição a conflitos parentais e vivência de punição física pela criança ou adolescente. Há ainda pais superprotetores que incentivam o comportamento violento dos filhos (Trautmann, 2008).

As pesquisas têm demonstrado uma associação entre a violência contra a criança (Grassi-Oliveira \& Stein, 2008; Marty \& Carvajal, 2005; Pinheiro \& Williams, 2009) e exposição à violência conjugal (Baldry, 2003; Pinheiro \& Williams, 2009; Weil et al., 2004) com o aparecimento de problemas de conduta dos adolescentes, como infração, comportamento antissocial e, em especial, bullying. Pinheiro e Williams (2009) verificaram em sua pesquisa que estar exposto à violência interparental esteve associado em ser alvo/autor de bullying na escola, sendo que a probabilidade de ser vítima de bullying foi 2,6 vezes maior para os estudantes expostos a qualquer tipo de violência por parte da mãe em relação àqueles que não a sofreram; e os alunos que declararam ter sofrido violência por parte do pai tinham 3,1 vezes mais chances de relatar também vitimização por bullying.

No mesmo sentido, Bauer et al. (2006) afirmam que um possível fator de risco para o bullying é a exposição à violência doméstica, pois crianças que passam por essa situação aprendem modelos de relacionamento negativos, no qual a violência é vista como método para resolução 
de conflitos. Além disso, tais crianças podem desenvolver mais problemas de comportamento inter e externalizantes, conforme definição de Achenbach (1991). Para Scholte et al. (2007), o bullying não é um fenômeno isolado, mas um componente de padrões comportamentais antissociais, que são construídos a partir do contato com diversos contextos, e aqui pode-se lembrar, ainda, a influência de aspectos econômicos, culturais e da mídia.

\section{Consequências imediatas do bullying}

O bullying pode ter consequências a curto e a longo prazo que dependem da frequência e intensidade do assédio, bem como das características da vítima, variando em relação ao impacto sobre diversas esferas da vida dos indivíduos. Na esfera emocional algumas consequências do bullying listadas na literatura são: problemas ou dificuldades sentimentais, medo, solidão e rebaixamento da autoestima (Batsche \& Knoff, 1994; Cepeda-Cuervo, Pacheco-Durán, García-Barco \& Piraquive-Penã, 2008; Lien et al., 2009; Lopes-Neto, 2005; Orpinas \& Horne, 2006; Spriggs et al., 2007; Whitted \& Dupper, 2008).

Em relação a problemas psiquiátricos, tem-se observado presença de ansiedade, depressão, ideação suicida ou tentativas de suicídio (Batsche \& Knoff, 1994; Campbell, 2004; Cepeda-Cuervo et al., 2008; Lamb et al., 2009; Lien et al., 2009; Orpinas \& Horne, 2006; Trautmann, 2008; Whitted \& Dupper, 2008). Há ainda a ocorrência de sintomas psicossomáticos, como dores de cabeça, dores de estômago, enurese, tonturas, problemas de sono e dores musculares (Campbell, 2004; Craig et al., 2009; Lamb et al., 2009; Lien et al., 2009; Orpinas \& Horne, 2006; Spriggs et al., 2007).

No que se refere aos problemas em relações interpessoais, constata-se a ocorrência de sentimento de ineficácia social e dificuldades de relacionamento interpessoal (Campbell, 2004; Scholte et al., 2007; Whitted \& Dupper, 2008). Podem estar presentes também comportamentos agressivos, homicídio a pares na escola, envolvimento com atividades ilícitas, como portar arma ou utilizar drogas (Batsche \& Knoff, 1994; Craig et al., 2009; Spriggs et al., 2007).

Além dos problemas típicos da vítima, os agressores podem apresentar também: baixo rendimento escolar, abandono da escola, envolvimento em condutas infracionais, problemas com o sistema legal e abuso de substâncias (Craig \& Pepler, 2003; Lamb et al., 2009; Orpinas \& Horne, 2006; Spriggs et al., 2007; Trautmann, 2008).

Hyman et al. (2003) alertam para as consequências graves de um ambiente escolar hostil, exemplificado, segundo tais autores, no relatório de Vossekuil, Fein, Reddy, Borum e Modzeleski (2002). Tal relatório, elaborado pelo Serviço Secreto dos Estados Unidos, examinou 37 incidentes envolvendo agressões fatais em escolas no mesmo país, entre 1974 e 2000, constatando que quase $3 / 4$ dos assassinos tinham sido vítimas de bullying, em alguns casos bullying severo e persistente, com um histórico de se sentirem ameaçados e perseguidos.

A realidade do aluno atirador dentro da escola não é apenas uma tendência norte-americana, tendo ocorrido em diversos países da Europa e da Ásia e, infelizmente, no Brasil. Williams (2004) descreve um caso que ocorreu em Taiúva, pequena cidade do interior de São Paulo, no ano de 2003, quando um jovem, com histórico de vitimização crônica na escola, feriu gravemente seis alunos, a vice-diretora, um funcionário e se suicidou. Posteriormente, em 2011, o jovem Wellington de 23 anos entrou atirando em sua antiga escola, em Realengo (no Rio de Janeiro), matando 11 estudantes, ferindo 13 e se suicidando em seguida (Brito, 2012). Para Lopes-Neto (2005), nos casos em que vítimas de bullying protagonizaram essas tragédias nas escolas, as agressões não tiveram alvos específicos, sugerindo que o desejo era de "matar a escola", local de intenso sofrimento e insegurança.

\section{Transtorno de Estresse Pós-Traumático e Bullying}

O conceito de trauma tem mudado ao longo do tempo (Schestatsky, Shansis, Ceitlin, Abreu, \& Hauck, 2003) e foge do escopo desse estudo o aprofundamento dessa questão. Adotar-se-á aqui o conceito de trauma propagado pela Associação Americana de Psiquiatria (2002) por ser o mesmo adotado pela Associação Americana de Psicologia e por psicólogos clínicos especializados da área. Nesse trabalho considera-se trauma como fenômeno resultante de dificuldades emocionais, comportamentais, cognitivas e físicas que são diretamente relacionadas a experiências violentas (Cohen, Mannarino, \& Deblinger, 2006). Experiências traumáticas são experiências prejudiciais e ameaçadoras que demandam esforços extraordinários por parte do indivíduo para enfrentamento ou sobrevivência, podendo, inclusive, favorecer o aparecimento de problemas psiquiátricos, como o Transtorno de Estresse Pós-Traumático (TEPT).

Segundo o DSM-IV-TR (Manual Diagnóstico Estatístico para os Transtornos Mentais, 2002), o TEPT tem como critérios de diagnóstico: A) Exposição a um evento traumático caracterizado por morte ou grave ferimento ou ameaça à integridade física própria ou de outros, e a resposta da pessoa envolveu medo intenso, impotência ou horror; B) O evento traumático é persistentemente revivido em uma ou mais das seguintes maneiras: recordações e sonhos aflitivos, recorrentes e intrusivos sobre o evento, flashbacks, sofrimento psicológico e reatividade fisiológica na exposição a indícios externos que lembram o evento traumático; C) Esquiva persistente de estímulos associados ao trauma e entorpecimento da reatividade geral, com base em pelo menos três dos seguintes quesitos: esforços para evitar pensamentos ou conversas associados ao trauma; evitação de atividades, locais e pessoas que ativem a recordação do trauma; incapacidade de recordar algum aspecto importante do evento; redução acentuada de interesse ou da participação em atividades significativas; sensação de distanciamento em relação a outros; afeto restrito e sentimento de um futuro abreviado; D) Sintomas persistentes de excitabilidade aumentada indicada por dois ou mais quesitos: dificuldades com o sono; irritabilidade ou surtos de raiva; dificuldade em concentrar-se; hipervigilância e resposta de sobressalto exagerada (Associação Americana de Psiquiatria, 2002).

É importante lembrar que para caracterizar TEPT, o quadro sintomático deve estar presente por pelo menos um mês e a perturbação deve causar sofrimento ou prejuízo significativo ao funcionamento social, ocupacional ou em 
outras áreas importantes da vida do indivíduo (Associação Americana de Psiquiatria, 2002).

Sobre o conceito de evento traumático, Figueira e Mendlowicz (2003) afirmam que ele tem sido constantemente revisto. Desde a oficialização do diagnóstico em 1980, houve uma expansão da latitude do conceito e, consequentemente, o aumento da prevalência estimada de TEPT. Tais autores mostram que, inicialmente, os eventos traumáticos eram classificados no DSM-III como raros, externos e catastróficos, diferenciados das experiências comuns de luto, doença crônica, conflitos matrimoniais e perdas no trabalho.

De acordo com Figueira e Mendlowicz (2003), a ênfase das pesquisas sobre TEPT em fatores externos poderia ser deslocada para os fatores internos ou subjetivos, de forma que a resposta emocional aos eventos traumáticos receba maior atenção. Elklit e Petersen (2008) afirmam que tanto a experiência clínica quanto as pesquisas têm fornecido evidências de que mesmo a exposição indireta a estressores pode favorecer o aparecimento de sintomas de TEPT, motivo pelo qual o critério de evento traumático foi expandido para incluir esse tipo de exposição indireta a diversos estressores.

Segundo a Associação Americana de Psiquiatria (2002), a prevalência de TEPT nos EUA é de $8 \%$ da população adulta, mas o distúrbio pode ocorrer em qualquer idade. Estima-se que cerca de $25 \%$ de todas as crianças experienciem um evento traumático até seus 16 anos, sendo que algumas apresentarão sintomas de TEPT (Cook-Cottone, 2004). De acordo com Marty e Carvajal (2005), um acontecimento traumático na infância seria capaz de produzir alterações funcionais e anatômicas em áreas cerebrais, modificando sistemas relacionados à memória e afetividade, entre outros e, assim, produzindo vulnerabilidade ao TEPT.

Para Lipschitz, Rasmusson, Anyan, Cromwell e Southwick (2000), a prevalência de TEPT indicada na literatura entre adolescentes e jovens adultos varia de $2 \%$ a $9,1 \%$. No estudo realizado por tais autores com 90 adolescentes do sexo feminino dos Estados Unidos, com idade média de 17,3 anos, 13\% apresentaram sintomas de TEPT, e a maioria foi exposta a múltiplos tipos de trauma. Segundo Elklit e Petersen (2008) e Weil et al. (2004), a exposição a múltiplos eventos traumáticos está associada ao aumento de sintomas de TEPT.

Estudos epidemiológicos revelaram que 40 a 90\% da população americana já foi exposta a um evento traumático (Figueira \& Mendlowicz, 2003). No Brasil ainda há carência de estudos sobre a sua prevalência, uma vez que somente recentemente a Psiquiatria brasileira começou a reconhecer a importância do diagnóstico de tal transtorno (Fiszman, Cabizuca, Lanfredi, \& Figueira, 2005); o mesmo ocorrendo em relação à Psicologia (Caminha, 2005). Para Figueira e Mendlowicz (2003) e Ximenes, Oliveira e Assis (2009), o TEPT é o principal transtorno psiquiátrico associado à violência, tendo uma alta prevalência entre a população brasileira.

Devido à importância do esclarecimento da relação entre a vivência de bullying e o desenvolvimento tardio de sintomas de TEPT, o presente trabalho objetivou identificar, organizar e analisar a produção cientifica da área quanto a esses dois temas, propondo direcionamentos para pesquisas futuras.

\section{Método}

Foi efetuada uma busca eletrônica de artigos indexados nas bases de dados SciELO, LILACS e PubMed no período de 2000 a 2012, com as seguintes palavras-chave "Bullying" e "Transtorno de Estresse Pós-Traumático" e "Bullying" e "Posttraumatic Stress Disorder". Tal busca resultou em 21 artigos, no entanto descartaram-se trabalhos que citavam TEPT, mas enfocavam outros temas, como Síndrome da Imunodeficiência Adquirida, psicose, bullying no trabalho e não entre pares escolares, entre refugiados e artigos opinativos, sem caráter empírico. Apenas dois artigos, dentre os 21, tratavam especificamente sobre bullying na escola durante a infância/adolescência e Transtorno de Estresse Pós-Traumático: Idsoe, Dyregrov e Idsoe (2012) e Elklit e Petersen (2008).

Além disso, foi realizado um levantamento bibliográfico em livros e artigos publicados sobre o tema, partindo-se de textos mais gerais sobre bullying e procurando-se nas referências, artigos que estivessem relacionados ao tema. Na busca bibliográfica foram encontrados três artigos específicos sobre bullying e TEPT na infância/adolescência: Crosby, Oehler e Capaccioli (2010), Mynard, Joseph e Alexander (2000) e Storch e Esposito (2003); e, finalmente, foram encontrados cinco estudos retrospectivos, examinando os sintomas de TEPT a longo prazo: Campbell (2004), Carlisle e Rofes (2007), Ateah e Cohen (2009), McGuckin Lewis, Cummins e Cruise (2011) e Williams, D’Affonseca, Correia e Albuquerque (2011). Apenas este último estudo é brasileiro, sendo que o mais antigo é de 12 anos atrás (Mynard et al., 2000).

\section{Resultados}

\section{Bullying e TEPT na infância e adolescência}

Dentre os estudos com população infantil e adolescente, está o de Elklit e Petersen (2008) que realizaram uma pesquisa com estudantes de quatro países europeus, com idade média de 14,5 anos, para investigar a prevalência de eventos traumáticos (como abuso, negligência e bullying) e o impacto psicológico desses eventos, inclusive o desenvolvimento de sintomas de TEPT. Os autores perceberam que 90\% dos adolescentes pesquisados reportaram ter experienciado diretamente ao menos um evento traumático, sendo que a média de exposição foi de 2,6 eventos. Dos participantes que sofreram um evento traumático, 25\% relataram ter sofrido humilhação ou bullying durante a infância.

Crosby et al. (2010) realizaram uma pesquisa com 244 estudantes, com idades entre 10 a 14 anos, de três escolas rurais dos Estados Unidos. Os autores buscaram dar suporte à hipótese de que a vitimização por pares/bullying pode estar associada a sintomas de TEPT. Por meio da análise dos questionários aplicados, os pesquisadores constataram que $13,5 \%$ dos participantes relataram ter sido vitimizados por um colega da escola pelo menos uma vez por semana, e $66 \%$ relataram ter presenciado alguém sofrer bullying ao menos uma vez no último ano. Vitimizações físicas, relacionais e verbais foram correlacionadas positivamente com sintomatologia de estresse pós-traumático. 
Com o objetivo de examinar se crianças que experienciaram bullying teriam altos níveis de sintomas de TEPT, Mynard et al. (2000) realizaram um estudo com 331 crianças e adolescentes, com idades entre 8 e 11 anos de escolas da Inglaterra e descobriram que experiências de vitimização por bullying estavam associadas a estresse pós-traumático. Os dados obtidos sugerem que cerca de um terço das crianças que sofreram bullying podem apresentar níveis significativos de estresse pós-traumático.

No mesmo sentido, Storch e Esposito (2003) realizaram uma pesquisa com o objetivo de examinar a relação entre vitimização por pares/bullying e TEPT numa amostra de 205 crianças, de 10 a 13 anos, dos Estados Unidos. Após análises estatísticas, os autores perceberam que dois tipos de bullying, vitimização direta e relacional, estavam relacionadas positivamente e significativamente aos sintomas de TEPT.

Idsoe et al. (2012) realizaram uma pesquisa com 963 estudantes de uma amostra representativa de crianças norueguesas das séries 8-9 (13 e 14 anos aproximadamente), para investigar a vitimização por bullying e associações com sintomas de TEPT. Como resultados principais, os autores constataram que havia uma associação entre a frequência de exposição a bullying e sintomatologia de TEPT, sendo que $33,7 \%$ dos estudantes que sofreram bullying apresentaram escores significativos para o transtorno. Segundo Idsoe et al. (2012), os resultados indicam que a exposição ao bullying é um potencial fator de risco para o desenvolvimento de sintomas de TEPT entre os estudantes vitimizados.

Os estudos apresentados demonstram que a exposição dos estudantes do Ensino Fundamental ao bullying em diferentes países é relativamente alta. Além disso, as pesquisas que relacionam bullying a sintomas de TEPT (Crosby et al., 2010; Mynard et al. 2000; Storch \& Esposito, 2003) indicaram correlações positivas e significativas entre os dois fenômenos, demonstrando que, a curto prazo, ser vítima de bullying pode levar ao aparecimento de sintomas de TEPT. Cabe destacar que, tanto Storch e Esposito (2003), quanto Mynard et al. (2000), afirmam que, apesar do aumento do número de pesquisas que investiguem as interações negativas entre pares, poucos estudos examinam a relação entre as consequências psicológicas dessas interações. Dentre tais interações destacam-se o TEPT e o bullying, o que dificulta que os autores comparem seus resultados (Mynard et al., 2000).

\section{Bullying e TEPT a longo prazo}

Como visto anteriormente, poucas pesquisas analisam a relação entre o bullying e as suas possíveis consequências traumáticas, sendo ainda mais escassa tal análise quanto às consequências traumáticas a longo prazo, que é o foco do presente estudo. Na pesquisa de Campbell (2004), a pesquisadora avaliou, retrospectivamente, a experiência de bullying na infância de 95 estudantes de graduação australianos. Os resultados indicaram um considerável número de vitimização praticada por pares $(68,8 \%)$, e também por professores $(21,2 \%)$ aos alunos; situações descritas como constrangedoras. No entanto, tal autora não chega a analisar os sintomas de TEPT propriamente ditos, tendo focado suas análises apenas na descrição das experiências de vitimização.
Carlisle e Rofes (2007) realizaram um estudo piloto com 15 adultos do sexo masculino, com histórico escolar de bullying. Ao medirem as consequências a longo prazo do bullying, os autores concluíram que seus efeitos eram mais amplos e persistentes do que o que já foi apontado pela literatura, sugerindo uma sintomatologia traumática semelhante a do histórico de abuso infantil, no qual o adulto é violento com a criança. Esses autores mencionam, por sua vez, que na ocasião do referido estudo foi encontrado apenas um trabalho quantitativo utilizando questionário específico, elaborado por Olweus (1993) que, apesar da amostra restrita (23 adultos), é citado pela literatura como evidência definitiva da vitimização, a longo prazo, por bullying. Carlisle e Rofes (2007) sugerem que se realizem mais pesquisas sobre os efeitos tardios de bullying, afirmando que alguns adultos que experienciaram bullying apresentam uma hiperexcitação crônica do Sistema Nervoso Central (altos níveis de medo, ansiedade e irritabilidade), sonhos intrusivos e relatam ter pensamentos relativos ao ambiente escolar, com teor de vingança, além de apresentarem dificuldades com figuras de autoridade.

De acordo com Ateah e Cohen (2009), casos severos de vitimização escolar podem preceder ou estar relacionados a TEPT, mostrando que muitas crianças correm o risco de experienciar o bullying de forma muito traumática, tendo consequências futuras relacionadas a esse tipo de vitimização. Os autores realizaram um estudo com 1.007 estudantes universitários dos Estados Unidos e 210 do Canadá, com o objetivo de avaliar o relacionamento entre bullying e a "pior experiência escolar" ao desenvolvimento de sintomas de TEPT. O resultado principal obtido foi que aproximadamente $8 \%$ da amostra canadense e $10 \%$ da americana alcançaram os critérios do DSM-IV para TEPT. Agressões verbais e relacionais foram as mais lembradas como piores experiências escolares, indicando que elas podem levar a profundos efeitos na saúde mental.

McGuckin et al. (2011) realizaram um estudo com 154 estudantes universitários da Irlanda, com idade média de 24,2 anos, investigando suas experiências escolares negativas passadas e os respectivos sintomas decorrentes por meio do questionário Student Alienation and Trauma Survey (SATS). Tais autores obtiveram como principal resultado que $6,2 \%$ da amostra apresentou escores clinicamente significativos para TEPT e $19,4 \%$ apresentou escores de risco.

No Brasil, Williams et al. (2011) realizaram um estudo piloto com 81 estudantes universitários, com idade média de 21 anos, utilizando uma versão traduzida para o português do questionário SATS, constatando que quase metade dos participantes (49\%) apresentou sintomas de TEPT após sua pior experiência escolar passada, sendo que 38\% desenvolveram um dos sintomas de TEPT por mais de um mês. Tais taxas altas em relação aos outros estudos provavelmente ocorreram porque o instrumento ainda necessitava de aprimoramento, pois conforme análise do índice de resposta inconsistente, $62 \%$ dos participantes respondeu o instrumento de tal forma. Posteriormente foi realizada uma adaptação transcultural do instrumento e atualmente uma pesquisa com amostra ampliada está sendo desenvolvida como Tese de Doutorado da primeira autora.

Apesar desses poucos estudos apontarem uma relação entre TEPT e bullying, tanto na infância quanto a longo 
prazo, faltam pesquisas na literatura nacional e internacional que esclareçam como se dá essa relação; esclarecendo, por exemplo, qual o tamanho da influência da vivência de bullying escolar no desenvolvimento futuro de sintomas de TEPT, considerando diversos outros eventos estressores nas vidas dos indivíduos. O que pode ser percebido é que alguns indivíduos expostos à vitimização por bullying podem apresentar maior vulnerabilidade para apresentar Transtorno de Estresse Pós-Traumático.

Como apontam Storch e Esposito (2003), componentes específicos que contribuem para TEPT, incluindo sentimentos de desamparo e descontrole, são inerentes à vitimização crônica e, por isso, é compreensível que esse tipo de vitimização, no caso a vivência de bullying, possa ser perigosa e ameaçadora suficientemente para produzir sintomas de TEPT. Isso se explicaria porque a vitimização repetitiva pode resultar em ruminações cognitivas e lembranças intrusivas, como sonhos e pensamentos sobre as interações negativas, evitação de situações associadas à vitimização e reatividade fisiológica. Considerando-se que os efeitos tardios do bullying, como o TEPT, ainda são pouco conhecidos e descritos pela literatura, são necessários mais estudos, principalmente longitudinais, para esclarecer a natureza desses relacionamentos.

\section{Conclusões}

Como apontado anteriormente, a exposição à violência pode ser uma experiência traumática que afeta, a curto e a longo-prazo, o bem-estar físico, social e emocional dos seres humanos. Dessa forma, devido ao impacto dos eventos traumáticos sobre a saúde mental dos indivíduos, essas experiências podem predispô-los tanto à resiliência quanto à vulnerabilidade (Miller, 2007).

A revisão bibliográfica realizada neste estudo apontou que parece existir uma relação entre a vivência de bullying escolar e o desenvolvimento de sintomas de TEPT. Dada a carência da literatura, ainda há dúvidas quanto aos efeitos a longo prazo do bullying; quais os tipos de agressões mais vivenciados pelos estudantes em estudos retrospectivos; que sintomas psiquiátricos, como os do TEPT, podem ser desenvolvidos por suas vítimas; bem como se a vivência de bullying escolar pode levar os indivíduos a sintomas atuais de TEPT ou eles já apresentam déficits psicológicos que predispõem o desenvolvimento de sintomas.

Pesquisas futuras são necessárias para responder às dúvidas existentes na área, dando consistência a dados esparsos de estudos científicos. Para tanto, seria preciso que tais trabalhos utilizassem diversos métodos de investigação, uma vez que todos os estudos aqui descritos usaram basicamente dados provenientes de questionários. Além disso, seria importante a coleta de dados com informantes variados, além dos próprios estudantes vitimizados, como pais e professores, por exemplo.

Análises mais sofisticadas da relação entre a vivência de bullying e os sintomas posteriores apresentados pelas vítimas poderiam esclarecer também o papel de variáveis moderadoras e mediadoras nesse processo. Nesse sentido, seriam interessantes estudos que avaliassem outros aspectos potencialmente traumáticos da vida dos indivíduos, como traumas no ambiente familiar, o que poderia esclarecer o quanto dos sintomas apresentados pelo indivíduo refere-se às experiências escolares traumáticas. Outras pesquisas também poderiam avaliar os efeitos dos fatores protetivos e de resilência presentes na época da escolarização, como por exemplo um sistema familiar de suporte, habilidades prossociais das crianças, as amizades, analisando o impacto de tais fatores para a proteção das crianças e jovens. Isso poderia ser investigado comparando-se grupos distintos de participantes, que possuem ou não situações de vulnerabilidade, por exemplo.

Com um corpo sólido de pesquisas apontando numa mesma direção, o intercâmbio entre os resultados de pesquisas diversas (que utilizam metodologias e instrumentos diferentes) seria facilitado, o que também possibilitaria o estabelecimento de conclusões sobre os impactos do bullying na saúde mental das vítimas. Além disso, tal corpo de conhecimento promoveria o estabelecimento de parâmetros de ação para os psicólogos que atendem caso de bullying, embasando, também, cursos para a sua prevenção nas escolas brasileiras, tanto para funcionários, professores, como para alunos. Futuros estudos poderão contribuir para o planejamento sistemático e eficaz de projetos de intervenção à violência na escola, bem como para o tratamento clínico de indivíduos que apresentem efeitos a longo prazo desse tipo de vitimização.

\section{Referências}

Achenbach, T. M. (1991). Integrative guide for the 1991 CBCL/418, YSR and TRF profiles. Burlington, VT: University of Vermont, Departament of Psychiatry.

Associação Americana de Psiquiatria. (2002). Manual Diagnóstico e Estatístico de Transtornos Mentais (DSM-IV-TR) (4a.ed.). Porto Alegre: Editora Artmed.

Ateah, A., \& Cohen, I. (2009). School victimization and bullying experiences: cross-national comparisons between Canada and the United States. New Scholarship in the Human Services, 8, 1-22.

Baldry, A. C. (2003). Bullying in schools and exposure to domestic violence. Child Abuse and Neglect, 27, 713-732.

Batsche, G. M., \& Knoff, H. M. (1994). Bullies and their victims: understanding a pervasive problem in the schools. School Psychological Review, 23, 165-175.

Bauer, N. S., Herrenkohl, T. I., Lozano, P., Rivara, F. P., Hill, K. G., \& Hawkins, J. D. (2006). Childhood bullying involvement and exposure to intimate partner violence. Pediatrics, 118 , 235-242.

Bond, L. Carlin, J. B., Thomas, L., Rubin, K., \& Patton, G. (2001). Does bullying cause emotional problems? A prospective study of young teenagers. British Medical Journal, 323, 480-484.

Brito, D. (2012). Alunos de Realengo tentam superar trauma um ano após o massacre. Folha de São Paulo. Disponível em: http:// www1.folha.uol.com.br/cotidiano/1072668-alunos-de-realengotentam-superar-trauma-um-ano-apos-o-massacre.shtml

Caminha, R. M. (2005). Transtornos do estresse pós-traumático (TEPT): da neurobiologia à terapia cognitiva. São Paulo: Casa do Psicólogo. 
Campbell, M. A. (2004). School victims: an analysis of 'my worst experience in school' scale. In E. McWilliam, S. Danby, \& J. Knight (Eds.), Performing Educational Research: Theories, Methods and Practices. (pp. 1-27). Flaxton, Australia: Post Pressed Flaxton.

Carlisle, N., \& Rofes, E. (2007). School bullying: do adult survivors perceive long-term effects? Traumatology, 13, 16-26.

Cepeda-Cuervo, E., Pacheco-Durán, P. N., García-Barco, L, \& Piraquive-Penã, C. J. (2008). Acoso escolar a estudiantes de educación básica y media. Revista de Salud Pública, 10, 517-528

Cerezo, F., \& Ato, M. (2010). Social status, gender, classroom climate and bullying among adolescents pupils. Anales de Psicologia, 26, 137-144.

Cohen, J. A., Mannarino, A. P., \& Deblinger, E. (2006). Treating trauma and traumatic grief in children and adolescents. New York: The Guilford Press.

Cook-Cottone, C. (2004). Childhood posttraumatic stress disorder: diagnosis, treatment, and school reintegration. School Psychology Review, 33, 127-139.

Craig, W., Harel-Fisch, Y., Fogel-Grinvald, H., Dostaler, S., Hetland, J., Simons-Morton, B., Molcho, M., Mato, M. G., Overpeck, M., Due, P., \& Picket, W. (2009). A cross-national profile of bullying and victimization among adolescents in 40 countries. International Journal of Public Health, 54, 216-224.

Craig, W. M., \& Pepler, D. J. (2003). Identifying and targeting risk for involvement in bullying and victimization. The Canadian Journal of Psychiatry, 48, 577-582.

Crosby, J. W., Oehler, J., \& Capaccioli, K. (2010). The relationship between peer victimization and post-traumatic stress symptomatology in a rural sample. Psychology in the Schools, 47, 297-310.

Elklit, A., \& Petersen, T. (2008). Exposure to traumatic events among adolescents in four nations. Torture, 18, 2-11.

Fekkes, M., Pijpers, F. I. M., Fridriks, M., Vogels, T., \& VerlooveVanhorick, S. P. (2010). Do bullied children get ill, or do ill children get bullied? A prospective cohort study on the relationship between bullying and health-related symptoms. Pediatrics, 117, 1568-1574.

Figueira, I., \& Mendlowicz, M. (2003). Diagnóstico do transtorno de estresse pós-traumático. Revista Brasileira de Psiquiatria, 25, 12-16.

Finkelhor, D., Turner, H. A., \& Hamby, S. (2012). Let's prevent peer victimization, not just bullying. Child Abuse and Neglect, 36, 271-274.

Fiszman, A., Cabizuca, M., Lanfredi, C., \& Figueira, I. (2005). A adaptação transcultural para o português do instrumento Trauma History Questionnaire para identificar experiências traumáticas. Revista Brasileira de Psiquiatria, 27, 63-66.

Francisco, M. V., \& Libório, R. M. C. (2009). Um estudo sobre bullying entre escolares do ensino fundamental. Psicologia: Reflexão e Crítica, 22, 200-207.

Furlong, M. J., Morrison, G. M., \& Jimerson, S. R. (2004). Externalizing behaviors of agression and violence and the school context. In Jr. R. B. Rutherford, M. M. Quinn \& S. R. Mathur (Eds.), Handbook of research in emotional and behavioral disorders (pp. 243-261). The Guilford Press: Nova York.

Grassi-Oliveira, R., \& Stein, L. M. (2008). Childhood maltreatment associated with PTSD and emotional distress in low-income adults: The burden of neglect. Child Abuse and Neglect, 32, 1089-1094.
Hyman, I., Kay, B., Mahon, M., Cohen, I., Tabori, A., Weber, M., Siegel, N., \& Glass, J. (2003). A cross national study of victimization of students: determining a typology of stressors and symptoms (Projeto de Pesquisa), National Center for the Study of Corporal Punishment and Alternatives, Temple University.

Idsoe, T., Dyregrov, A., \& Idsoe, E. C. (2012). Bullying and PTSD symptoms. Journal of Abnormal Child Psychology, 40, 901-911.

Lamb, J., Pepler, D. J., \& Craig, W. (2009). Approach to bullying and victimization. Canadian Family Physician, 55, 356-360.

Lien, L., Green, K., Welander-Vatn, A., \& Bjertness, E. (2009). Mental and somatic health complaints associated with school bullying between 10 e 12 grade students: results from cross sectional studies in Oslo, Norway. Clinical Practice and Epidemiology in Mental Health, 5, 1-8.

Lipschitz, D. S., Rasmusson, A. M., Anyan, W., Cromwell, P., \& Southwick, S. M. (2000). Clinical and functional correlates of posttraumatic stress disorder in urban adolescent girls at a primary care clinic. Journal of the American Academy of Child and Adolescent Psychiatry, 39, 1104-1111.

Lopes Neto, A. A., \& Saavedra, L. H. (2003). Diga não para o bullying: programa de redução do comportamento agressivo entre estudantes. Rio de Janeiro: ABRAPIA.

Lopes-Neto, A. A. (2005). Bullying - comportamento agressivo entre estudantes. Jornal de Pediatria, 81, 164-172.

Marty, C. M. \& Carvajal, C. A. (2005). Maltrato infantil como factor de riesgo de transtorno por estres postraumático en la adultez. Revista Chilena de Neuro-Psiquiatría, 43, 180-187.

McGuckin, C., Lewis, C. A., Cummins, P. K., \& Cruise, S. M. (2011). The stress and trauma of school victimization in Ireland: A retrospective account. Psychology, Society \& Education, 3, 55-67.

Miller, T. W. (2007). Trauma, change, and psychological health in the 21st Century. American Psychologist, 62, 889-898.

Mynard, H., Joseph, S., Alexander, J. (2000). Peer-victimization and posttraumatic stress in adolescents. Personality and Individual Differences, 29, 815-821.

Neme, C. M. B., Mello, L. C. de, Gazzola, R. A., \& Justi, M. M. (2008). Fenômeno bullying: análise de pesquisas em Psicologia publicadas no período de 2000 a 2006. Pediatria Moderna, 4, 200-204.

Olweus, D. (1991). Bully/victim problems among school children: Basic facts and effects of a school based intervention program. In D. Pepler \& K. Rubin (Eds.), The development and treatment of childhood aggression (pp 411-448). Hillsdale, NJ: Erlbaum.

Olweus, D. (1993). Bullying at school: what we know and what we can do. Oxford: Blackwell Publishers.

Orpinas, P., \& Horne, A. M. (2006). Bullies and victims: a challenge for schools. In J. R. Lutzker (Ed.), Preventing violence: research and evidence-based intervention strategies (pp. 147-165). Washington: American Psychological Association.

Pinheiro, F. M. F., \& Williams, L. C.A. (2009). Violência intrafamiliar e intimidação entre colegas no Ensino Fundamental. Cadernos de Pesquisa, 39, 995-1018.

Ruotti, C., Alves, R., \& Cubas, V. O. (2007). Violência na escola: um guia para pais e professores. São Paulo: Andhep: Imprensa Oficial do Estado de São Paulo.

Schestatsky, S., Shansis, F., Ceitlin, L. H. Abreu, P. B. S. \& Hauck, S. (2003). A evolução histórica do conceito de estresse póstraumático. Revista Brasileira de Psiquiatria, 25, 8-11. 
Scholte, R. H. J., Engels, R. C. M. E., Overbeek, G., Kemp, R. A. T. de., \& Haselager, G. J. T. (2007). Stability in bullying and victimization and its association with social adjustment in childhood and adolescence. Journal of Abnormal Child Psychology, 37, 217-228.

Spriggs, M. A., Iannotti, R. J., Nansel, T. R., \& Haynie, D. L. (2007). Adolescent bullying involvement and perceived family, peer and school relations: commonalities and differences across race/ethnicity. Journal of Adolescent Health, 41, 283-293.

Storch, E. A., Esposito, L. E. (2003). Peer victimization and posttraumatic stress among children. Child Study Journal, 33, 91-98.

Trautmann, A. M. (2008). Maltrato entre pares o "bullying". Una vision actual. Revista Chilena de Pediatria, 79, 13-20.

Vossekuil, B., Fein, R., Reddy, M., Borum, R., \& Modzeleski, W. (2002). The final report and findings of the Safe school initiative: Implications for the prevention of school attacks in the United States. Washington: United States Secret Service and United States Department of Education.

Weil, K. P., Florenzano, R. U., Vitriol, V. G., Cruz, C. M., Carvajal, C.A., Fullerton, C. U., \& Muñiz, C. D. (2004). Trauma infanto juvenil y psicopatología adulta: Un estudio empírico. Revista Médica de Chile, 132, 1499-1504.
Whitted, K. S., \& Dupper, D. R. (2008). Do teachers bully students? Findings from a survey of students in an alternative education setting. Education and Urban Society, 40, 329-341.

Williams, L. C.A. (2004). Violência e suas diferentes representações. In: G. C. Solfa, (Ed.), Gerando cidadania: Reflexões, propostas e construções práticas sobre direitos da criança e do adolescente. (pp. 141-153). São Carlos: Rima.

Williams, L. C. A., D'Affonseca, S. M., Correia, T. A., \& Albuquerque, P. P. (2011). Efeitos a longo prazo da vitimização escolar. Gerais: Revista Interinstitucional de Psicologia, 4, 187-199.

Ximenes, L. F., Oliveira, R. V. C., \& Assis, S. G. (2009). Violência e transtorno de estresse pós-traumático na infância. Ciência e Saúde Coletiva, 14, 417-433.

Recebido em 26.04.2010 Primeira decisão editorial em 17.09.2012 Versão final em 18.01.2013 Aceito em 14.02.2013

\section{Adoção e Acolhimento Institucional}

Santo André, SP, BR

25 de Fevereiro de 2013 